\title{
Lattice distortion induced first- and second-order topological phase transition in a rectangular high- $T_{c}$ superconducting monolayer
}

\author{
Li Chen $\odot,{ }^{1,2,3, *}$ Bin Liu, $1,2, *$ Gang Xu, ${ }^{2,1,3, \dagger}$ and Xin Liu ${ }^{1,2,3, *}$ \\ ${ }^{1}$ School of Physics, Huazhong University of Science and Technology, Wuhan, Hubei 430074, China \\ ${ }^{2}$ Wuhan National High Magnetic Field Center, Huazhong University of Science and Technology, Wuhan, Hubei 430074, China \\ ${ }^{3}$ Institute for Quantum Science and Engineering, Huazhong University of Science and Technology, Wuhan, Hubei 430074, China
}

(Received 21 October 2019; revised 3 May 2021; accepted 4 May 2021; published 1 June 2021)

\begin{abstract}
We theoretically study the lattice distortion induced first- and second-order topological phase transition in rectangular $\mathrm{FeSe}_{x} \mathrm{Te}_{1-x}$ monolayer. When compressing the lattice constant in one direction, our first-principles calculation shows that the $\mathrm{FeSe}_{x} \mathrm{Te}_{1-x}$ undergoes a band inversion at $\Gamma$ point in a wide doping range, say $x \in(0.0,0.7)$, which indicates the first-order topological phase transition of its electronic band structure. Meanwhile, because the unidirectional pressure breaks $\mathrm{C}_{4}$ symmetry, the topological edge states along the (100) and (010) directions have different Dirac energy. Given the high-temperature superconductivity of $\mathrm{FeSe}_{x} \mathrm{Te}_{1-x}$ monolayer, we found that the $\mathrm{C}_{4}$ symmetry breaking is essential to support Majorana corner states in either presence or absence of time-reversal symmetry. Especially in the case of breaking time-reversal symmetry, we can obtain a single Majorana zero mode at each corner without concerning the details of the superconducting pairing symmetries and applied Zeeman form, which can potentially bring advantages in the experimental implementation.
\end{abstract}

DOI: 10.1103/PhysRevResearch.3.023166

\section{INTRODUCTION}

The hybrid of superconductivity and topological band structure can provide an experimentally accessible platform to obtain the Majorana zero modes (MZMs) [1]. In the early studies [1-7], this hybrid is achieved in the superconductor/topological insulator heterostructure due to the superconducting proximity effect. The proximity-induced superconducting gap is sensitive to the interface of the heterostructure and normally one order of magnitude smaller than the gap in the mother superconductor, which brings various difficulties in the experimental verification of MZMs. Thus, it is essential to realize the MZMs in a large-gap superconductor without a complex heterostructure. On the other hand, iron-based superconductor was originally discovered as the first fully gapped high- $T_{c}$ superconductor, which has multibands at the Fermi level [8-14]. Recent studies show that the existence of multibands at the Fermi level is helpful for the coexistence of high- $T_{c}$ superconductivity and topological band structure in one material without the complex heterostructure [15-18]. For example, following the theoretical prediction $[19,20]$, the zero-bias conductance peak has been

\footnotetext{
${ }^{*}$ These authors contributed equally to this work.

†gangxu@hust.edu.cn

‡phyliuxin@hust.edu.cn
}

Published by the American Physical Society under the terms of the Creative Commons Attribution 4.0 International license. Further distribution of this work must maintain attribution to the author(s) and the published article's title, journal citation, and DOI. observed at the surface vertex core [16,21-23]. Meanwhile, the theoretical studies of high-order topological superconductors [24-48] provide new insight to realize the MZMs directly from two-dimensional and three-dimensional systems without breaking the uniformity of the bulk Hamiltonian. Thus, the implementation of the high-order topological superconductors in iron-based superconductors can provide a promising approach to achieve the MZMs in one large-gap superconductor and avoiding complex heterostructure.

In this paper, we theoretically propose to implement a first-order topological band structure and a secondorder topological superconductor in a monolayer rectangular $\mathrm{FeSe}_{x} \mathrm{Te}_{1-x} / \mathrm{SrTiO}_{3}(110)$, as shown in Fig. 1. Our firstprinciples calculations show that when the in-plane lattice constant in one direction is reduced, the electronic band structure of the monolayer $\mathrm{FeSe}_{x} \mathrm{Te}_{1-x}$ can achieve a strong TI phase within a wide range of composition $x$ values. This results in the first-order topological phase transition (TPT) of the inverted band structures with one pair of helical edge states at each edge. As the $\mathrm{FeSe}_{x} \mathrm{Te}_{1-x} / \mathrm{SrTiO}_{3}(110)$ monolayer has been experimentally observed to be a high- $T_{c}$ superconductor [49], we further study its topological property in the presence of the superconductivity. Note that the symmetry of the system is broken down to $\mathrm{D}_{2 \mathrm{~h}}$ due to reducing the lattice constant in one direction, the edge states along (100) and (010) direction have different electronic Dirac energies. When applying either only the superconducting potential or together with the in-plane magnetic field, the edge states along the two directions are naturally in different gapped phases. Thus the $\mathrm{C}_{4}$ symmetry breaking is very helpful for the implementation of Majorana corner states in both time-reversal invariant and breaking monolayer $\mathrm{FeSe}_{x} \mathrm{Te}_{1-x}$. In particular, our results for 


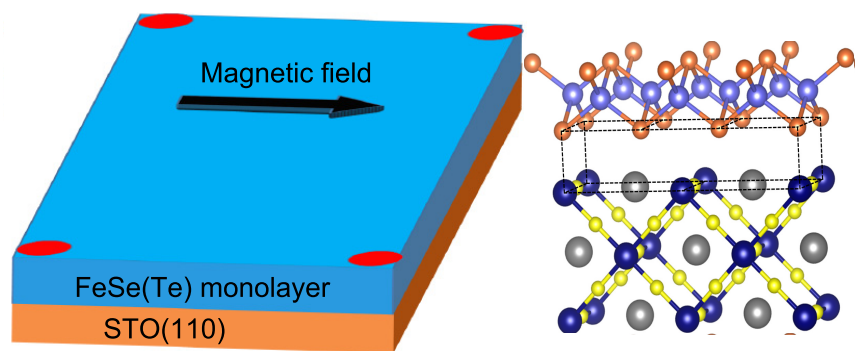

FIG. 1. Experimental set up of $\mathrm{FeSe}_{x} \mathrm{Te}_{1-x} / \mathrm{SrTiO}_{3}$ (110) monolayer. The red points indicate the existence of Majorana corner states.

D class monolayer rectangular $\mathrm{FeSe}_{x} \mathrm{Te}_{1-x} / \mathrm{SrTiO}_{3}$ do not depend on the exact superconducting pairing symmetries $(s-$ wave pairing, $s_{ \pm}$pairing) and the details of the Zeeman form, and are robust against disorders, which give the rectangle monolayer $\mathrm{FeSe}_{x} \mathrm{Te}_{1-x} / \mathrm{SrTiO}_{3}$ great advantages in experimentally achieving MZMs.

This paper is organized as follows. In Sec. II, we study the first-order topological phase transition of the electronic band structures. In Sec. II A, we perform our first-principles calculations to confirm the topological band inversion assisted by the lattice distortion. In Sec. II, according to our firstprinciples calculations, we construct the model Hamiltonian, which is used to obtain the edge Hamiltonian and study the edge states. In Sec. III, based on the first-order topological phase transition of the electronic band structure, we study the second-order topological phase transition of the DIII and D classes in Sec. III(a) and Sec .III(b), respectively. In Sec. IV, we discuss the experimental reality for our model and give the conclusion.

\section{LATTICE DISTORTION INDUCED FIRST-ORDER TPT}

\section{A. The first-principles calculations of the band structure}

The first-principles calculations based on densityfunctional theory [50,51] (DFT) are performed to study the topological band structure of the monolayer $\mathrm{FeSe}_{x} \mathrm{Te}_{1-x}$ with $\mathrm{C}_{4}$ symmetry breaking. The system initially has square lattice with lattice constant $a_{0}=3.905 \AA$, which is the same with the (001) monolayer $\mathrm{FeSe}_{x} \mathrm{Te}_{1-x} / \mathrm{SrTiO}_{3}$ [18]. Without losing the generality, we consider compressing the lattice constant in [100] direction, quantified by the ratio $\epsilon=\left(a_{0}-a_{x}\right) / a_{0}$ with $a_{x}$ the lattice constant along $x$ direction after compression. In this case, the states at the $\Gamma$ point are classified as the $D_{2 h}$ representations. Near Fermi level, we consider four orbital states, the odd parity $B_{1 u}$ states contributed by $\mathrm{Se}(\mathrm{Te}) p_{z}$ orbital and the even parity $A_{g}, B_{2 g}$, and $B_{3 g}$ states mainly coming from $\mathrm{Fe} d_{x^{2}-y^{2}}, d_{x z}$, and $d_{y z}$ orbitals, respectively. We distinguish the parities by green and blue dots in Fig. 2(a). Note that the $d_{x z}$ and $d_{y z}$ orbitals are not degenerate anymore due to the $\mathrm{C}_{4}$ symmetry breaking. We first choose the typical composition value $x=0.45$ [16] for which the monolayer $\mathrm{FeSe}_{x} \mathrm{Te}_{1-x} / \mathrm{SrTiO}_{3}$ with $\mathrm{C}_{4}$ symmetry is in topological trivial phase [18]. The calculated band structures for $\epsilon=2 \%$ and $\epsilon=6 \%$ are plotted in Figs. 2(a) and 2(b), respectively, in which red and black curves correspond to the band spectrum with and without spin-orbital coupling (SOC). For $\epsilon=2 \%$ (a)
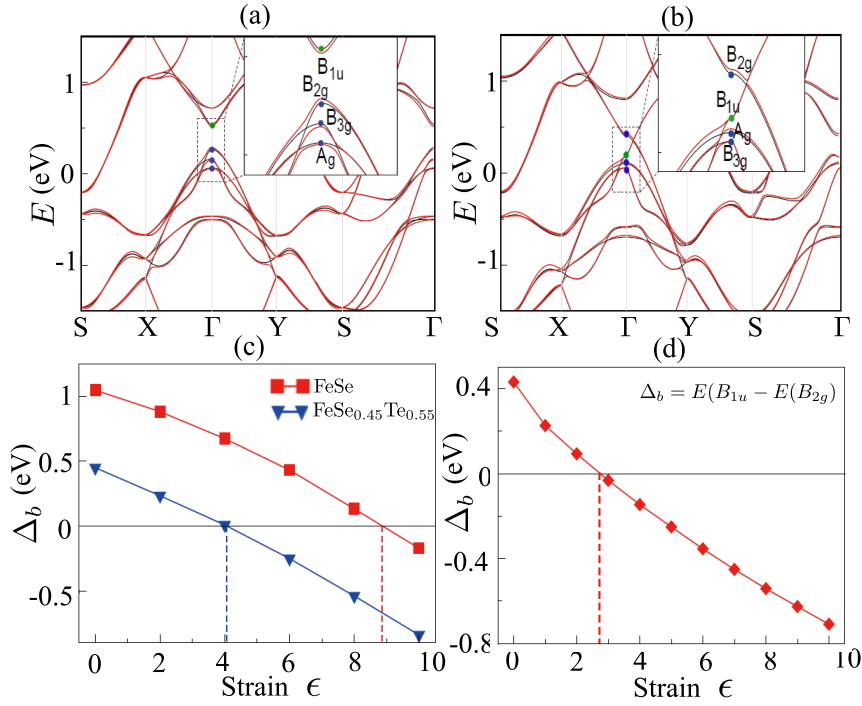

FIG. 2. (a) Band structure of $\mathrm{FeSe}_{0.45} \mathrm{Te}_{0.55}$ with the lattice distortion $\epsilon=2 \%$. (b) Band structure of $\mathrm{FeSe}_{0.45} \mathrm{Te}_{0.55}$ with the lattice distortion $\epsilon=6 \%$. (c) The band gap $\Delta_{b}$ of $\mathrm{FeSe}_{x} \mathrm{Te}_{1-x}$ monolayer as the function of distortion $\epsilon$ with $x=1$ (red lines) and $x=0.45$ (blue lines). (d) The band gap $\Delta_{b}$ of $\mathrm{FeSe}_{x} \mathrm{Te}_{1-x}$ monolayer as a function of Te concentration $1-x$ with $\epsilon=6 \%$.

shown in Fig. 2(a), there is no band inversion so that the system is in topological trivial phase and has a positive band gap at $\Gamma$ point $\Delta_{\mathrm{b}}=E\left(B_{1 u}\right)-E\left(B_{2 g}\right)>0$. For the bands of $\epsilon=6 \%$ shown in Fig. 2(b), band inversion happens between the odd parity state $B_{1 u}$ and even parity state $B_{2 g}$ at $\Gamma$ point, and has a negative $\Delta_{\mathrm{b}}<0$. When SOC is excluded [see the black bands in Fig. 2(b)], this band inversion leads to a linear band crossing between $B_{1 u}$ and $B_{2 g}$ states only along $\Gamma-Y$ due to the $\mathrm{C}_{4}$ symmetry breaking. When considering SOC, the linear crossing is opened, resulting in a topologically nontrivial band gap so that the system falls into a 2D TI phase around $\Gamma$ point. Our first-principles calculations (Appendix B) show that the size of the SOC-induced gap is the same order as that in 3D FeSeTe [17,20]. Thus the anisotropic lattice distortion, by increasing the compress ratio, induces the first order TPT. In Fig. 2(c), we plot the band gap $\Delta_{\mathrm{b}}$ as a function of the compress ratio $\epsilon$ increasing from 0 to $10 \%$, for different compositions $x=1$ (red square for FeSe) and $x=0.45$ (blue triangle for $\mathrm{FeSe}_{0.45} \mathrm{Te}_{0.55}$ ), respectively. The calculated results show that both systems are in the trivial phase for $\epsilon=0$ [18]. With increasing the compression, the band gap $\Delta_{\mathrm{b}}$ undergoes a sign change at the critical value $\epsilon_{\mathrm{c}}=9 \%$ and $\epsilon_{\mathrm{c}}=4 \%$ [indicated by red and blue dashed lines in Fig. 2(c)], for $\mathrm{FeSe}$ and $\mathrm{FeSe}_{0.45} \mathrm{Te}_{0.55}$, respectively. As reported by Ref. [49], high- $T_{c}$ superconductivity has already been observed in $\mathrm{FeSe} / \mathrm{SrTiO}_{3}(110)$ with the rectangular lattice [49]. The $\mathrm{SrTiO}_{3}(110)$ has the lattice constants $a=3.905 \AA$ and $b=\sqrt{2} a$. This lattice mismatch makes three unit cells of FeSe grow on the top of two STO(110) unit cells, which reduces the FeSe lattice constant in [100] direction to $\frac{2}{3} \sqrt{2} a \approx 0.94 a$, corresponding to $\epsilon=6 \%$ in our calculations. This growth technique should also be applied to $\mathrm{FeSe}_{x} \mathrm{Te}_{1-x}$ monolayer. Therefore considering the experimental reality, we take $\epsilon=6 \%$ and plot the band gap $\Delta_{\mathrm{b}}$ as a function of the Te 

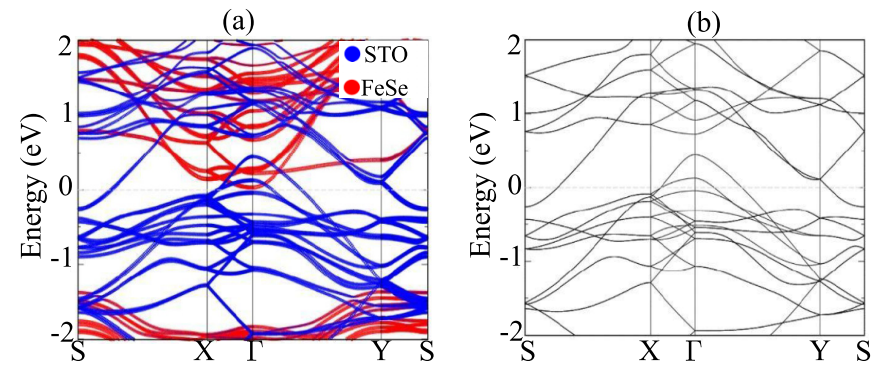

FIG. 3. The energy bands for (a) the monolayer FeSe on 5 layers of $\mathrm{SrTiO}_{3}$ (110) substrate and (b) the free-standing rectangular FeSe monolayer. The red and blue curves in (a) are the bands from $\mathrm{SrTiO}_{3}$ and FeSe, respectively. In both figures, the Fermi level is set at $0 \mathrm{eV}$ as the gray dashes.

composition in Fig. 2(d). In order to see the influence of STO on FeSe, we perform more realistic calculations by putting the monolayer FeSe on 5 layers of STO(110) substrate, where the distance is set as $2 \AA$, very similar to the previous study [52]. The calculated band structures are plotted in Fig. 3(a), which shows that the electronic states from $\mathrm{SrTiO}_{3}$ (red curves) are almost decoupled with those from FeSe (blue curves). In Fig. 3(b), we also plot the energy bands of the free-standing rectangular FeSe monolayer without $\mathrm{SrTiO}_{3}$ substrate. Comparing the FeSe bands in the two figures shows that there is little difference between them. Therefore, the substrate $\mathrm{SrTiO}_{3}(110)$ has little effect on FeSe monolayer except for distorting the FeSe lattice constant. These results strongly suggest that both the superconductivity and topological band structures could coexist in the rectangular lattice $\mathrm{FeSe}_{x} \mathrm{Te}_{1-x}$ for $0.3<x<0.7[18,49,53]$.

\section{B. The model Hamiltonian and the edge theory}

To further investigate the topological edge states, we construct an eight-bands $k \cdot p$ effective model Hamiltonian with $D_{2 h}$ symmetry. The basis of the rectangular FeSeTe monolayer from the bonding and antibonding states are taken as

$$
\begin{aligned}
& \left|P_{\alpha}^{ \pm}\right\rangle=\frac{1}{\sqrt{2}}\left(\left|\mathrm{Se}^{\mathrm{A}}, \mathrm{p}_{\alpha}\right\rangle \mp\left|\mathrm{Se}^{\mathrm{B}}, \mathrm{p}_{\alpha}\right\rangle\right), \\
& \left|D_{\beta}^{ \pm}\right\rangle=\frac{1}{\sqrt{2}}\left(\left|\mathrm{Fe}^{\mathrm{A}}, \mathrm{d}_{\beta}\right\rangle \pm\left|\mathrm{Fe}^{\mathrm{B}}, \mathrm{d}_{\beta}\right\rangle\right),
\end{aligned}
$$

where $\mathrm{A}$ and $\mathrm{B}$ are the two sublattices of $\mathrm{Fe}$ and $\mathrm{Se}(\mathrm{Te})$ atoms, $\alpha=(x, y, z)$ and $\beta=\left(x z, y z, x y, x^{2}-y^{2}, z^{2}\right)$ are the $4 p$ and $3 d$ orbitals of Se and Fe, respectively. The superscript $+(-)$ indicates the even (odd) parity of the states. According to the DFT calculations, the states around the Fermi surface at $\Gamma$ point mainly comes from $\left|D_{y z}^{+}\right\rangle,\left|D_{x^{2}-y^{2}}^{+}\right\rangle,\left|D_{x z}^{+}\right\rangle$and $\left|P_{z}^{-}\right\rangle$. Because of the $C_{4}$ symmetry breaking, the effective Hamiltonian at $\Gamma$ point has the point group symmetry $D_{2 h}$, whose generators include inversion $I$, rotations around $z$-axis $R_{2 z}$ and $y$-axis $R_{2 y}$. This provides the constraint on the electronic Hamiltonian

$$
R H_{e}(\boldsymbol{k}) R^{-1}=H_{e}(R \boldsymbol{k}),
$$

where $R$ denotes the symmetry operators in $D_{2 h}$ group. This leads the electronic Hamiltonian, up to the second-order of $k$
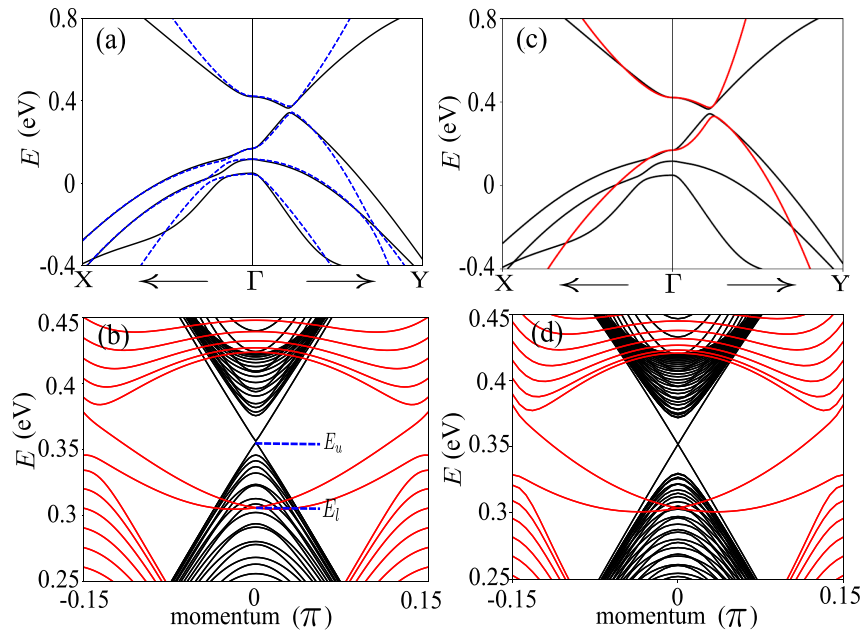

FIG. 4. (a) Fitting the band dispersions (black) for $\epsilon=6 \%$ with eight bands $k \cdot p$ Hamiltonian $H_{e}$ (blue dash). (b) Band dispersions of $k_{x}$ (black) and $k_{y}$ (red) for eight bands model $H_{e}$. (c) Fitting the band dispersions (black) with projected four bands Hamiltonian $H_{\text {eff }}$ (red curves). (d) Band dispersions of $k_{x}$ (black) and $k_{y}$ (red) for projected four bands Hamiltonian $H_{\mathrm{eff}}$.

around $\Gamma$ point is obtained as $H_{\mathrm{e}}=H_{0}(k)+H_{\text {soc }}$. Here

$$
H_{0}=\left(\begin{array}{cccc}
M_{1}(\mathbf{k}) & 0 & \beta k_{x} k_{y} & i \gamma k_{y} \\
0 & M_{2}(\mathbf{k}) & 0 & 0 \\
\beta k_{x} k_{y} & 0 & M_{3}(\mathbf{k}) & i \delta k_{x} \\
-i \gamma k_{y} & 0 & -i \delta k_{x} & M_{4}(\mathbf{k})
\end{array}\right)
$$

is the Hamiltonian without spin-orbit coupling (SOC), with $M_{i}(k)=E_{i}+M_{i x} k_{x}^{2}+M_{i y} k_{y}^{2}(i=1,2,3,4), E_{i}$ the energy of the $i$ th doubly degenerated band at $\Gamma$ point, $M_{i x}$ and $M_{i y}$ the in-plane hopping of band $i, \gamma$, and $\delta$ the interband hopping magnitude. $C_{4}$ symmetry breaking makes $M_{i x} \neq M_{i y}$ and $\gamma \neq \delta$.

$H_{\mathrm{soc}}=\left(\begin{array}{cccccccc}0 & 0 & i \lambda_{1} & 0 & 0 & -i \lambda_{3} & 0 & 0 \\ 0 & 0 & 0 & 0 & i \lambda_{3} & 0 & \lambda_{2} & 0 \\ -i \lambda_{1} & 0 & 0 & 0 & 0 & -\lambda_{2} & 0 & 0 \\ 0 & 0 & 0 & 0 & 0 & 0 & 0 & 0 \\ 0 & -i \lambda_{3} & 0 & 0 & 0 & 0 & -i \lambda_{1} & 0 \\ i \lambda_{3} & 0 & -\lambda_{2} & 0 & 0 & 0 & 0 & 0 \\ 0 & \lambda_{2} & 0 & 0 & i \lambda_{1} & 0 & 0 & 0 \\ 0 & 0 & 0 & 0 & 0 & 0 & 0 & 0\end{array}\right)$

is the spin-orbit Hamiltonian in the basis $(|\uparrow\rangle,|\downarrow\rangle) \otimes\left(\left|D_{y z}^{+}\right\rangle\right.$, $\left|D_{x^{2}-y^{2}}^{+}\right\rangle,\left|D_{x z}^{+}\right\rangle$and $\left.\left|P_{z}^{-}\right\rangle\right)$, with $\lambda_{1}, \lambda_{2}$, and $\lambda_{3}$ are the SOC amplitudes. The fitting parameters of the 8-bands effective model with DFT calculations are shown in Table I.

In Fig. 4(a), we show that with the fitting parameters, our model can describe the band dispersion near $\Gamma$ point well. With these parameters, in Fig. 4(b) we plot the band dispersions for the system in the slab geometry with the open boundary along $y$ (black curves) and $x$ (red curves), respectively. The Dirac points of these two edges have different energies with $E_{u}=0.36 \mathrm{eV}$ (upper Dirac point) and $E_{l}=0.31 \mathrm{eV}$ (lower Dirac point). This results in the Dirac energy difference $\delta E_{\mathrm{D}}$, about $50 \mathrm{meV}$, which is consistent with the first-principles calculations (Appendix B). 
TABLE I. Fitting parameters of eight bands model Hamiltonian.

\begin{tabular}{lcccccccc}
\hline \hline$E_{1} / \mathrm{eV}$ & $E_{2} / \mathrm{eV}$ & $E_{3} / \mathrm{eV}$ & $E_{4} / \mathrm{eV}$ & $\beta\left(\mathrm{eV} \AA^{2}\right)$ & $M_{1 x}\left(\mathrm{eV} \AA^{2}\right)$ & $M_{1 y}\left(\mathrm{eV} \AA^{2}\right)$ & $M_{2 x}\left(\mathrm{eV} \AA^{2}\right)$ & $M_{2 y}\left(\mathrm{eV} \AA^{2}\right)$ \\
\hline 0.048 & 0.115 & 0.42 & 0.168 & 1.05 & -1.9 & -3.6 & -1.6 \\
$\lambda_{1} / \mathrm{eV}$ & $\lambda_{2} / \mathrm{eV}$ & $\lambda_{3} / \mathrm{eV}$ & $\delta(\mathrm{eV} \AA)$ & $\gamma(\mathrm{eV} \AA)$ & $M_{3 x}\left(\mathrm{eV} \AA^{2}\right)$ & $M_{3 y}\left(\mathrm{eV} \AA^{2}\right)$ & $M_{4 x}\left(\mathrm{eV} \AA^{2}\right)$ & $M_{4 y}\left(\mathrm{eV} \AA^{2}\right)$ \\
\hline 0.024 & 0.017 & 0.014 & 2.1 & 1.95 & -2.9 & -4.4 & 3.4 & 4.1 \\
\hline \hline
\end{tabular}

The anisotropy of the electronic Dirac point energies is due to the $\mathrm{C}_{4}$ symmetry breaking by lattice distortion. As this is essential for realizing the second-order topological phase transition, we construct the edge theory to study the effect of the lattice distortion on the edge states. As shown in Fig. 2(b), the band inversion happens between $\left|P_{z}^{-}\right\rangle$and $\left|D_{x z}^{+}\right\rangle$ at $\Gamma$ point. These two bands cross along $\Gamma-Y$ while the other bands are far away from the crossing point. So we can project the original eight bands model into these two orbitals to obtain a simplified four bands Hamiltonian. For the convenience of the projection, we switch the basis order to $\left(\left|D_{x z}^{+}, \uparrow\right\rangle\right.$, $\left|P_{z}^{-}, \uparrow\right\rangle,\left|D_{x z}^{+}, \downarrow\right\rangle,\left|P_{z}^{-}, \downarrow\right\rangle,\left|D_{y z}^{+}, \uparrow\right\rangle,\left|D_{x^{2}-y^{2}}^{+}, \uparrow\right\rangle,\left|D_{y z}^{+}, \downarrow\right\rangle$, $\left.\left|D_{x^{2}-y^{2}}^{+}, \downarrow\right\rangle\right)$ so that the eight bands full Hamiltonian takes the form [54]

$$
\begin{gathered}
H_{e}(k) \equiv \epsilon+\mathbb{H}_{0}+\mathbb{H}_{\text {non }}, \\
\mathbb{H}_{0}=\left(\begin{array}{cc}
H_{\pi}-\epsilon & 0 \\
0 & H_{\sigma}-\epsilon
\end{array}\right), \quad \mathbb{H}_{\text {non }}=\left(\begin{array}{cc}
0 & H_{c} \\
H_{c}^{\dagger} & 0
\end{array}\right),
\end{gathered}
$$

where $H_{\pi}, H_{\sigma}$ and $H_{c}$ are $4 \times 4$ matrices. The eigenvalues of $H_{\pi}$ are around energy $\epsilon$ where the eigenvalues of $H_{\sigma}$ are away from $\epsilon$. Up to second order, the projected four bands Hamiltonian $H_{\text {eff }}$ can be written as

$$
H_{\mathrm{eff}} \simeq H_{\pi}-H_{c}\left(H_{\sigma}-\epsilon\right)^{-1} H_{c}^{\dagger} .
$$

Then we obtain

$$
H_{\mathrm{eff}}=\left(\begin{array}{cccc}
M_{3}+\delta_{1} & i \eta k_{x}+\gamma k_{y} & 0 & 0 \\
-i \eta k_{x}+\gamma^{*} k_{y} & M_{4}+\delta_{2} & 0 & 0 \\
0 & 0 & M_{3}+\delta_{1} & i \eta k_{x}-\gamma^{*} k_{y} \\
0 & 0 & -i \eta k_{x}-\gamma k_{y} & M_{4}+\delta_{2}
\end{array}\right)
$$

We label the four edges of a rectangular as I, II, III, and IV [Fig. 5(a)], and consider edge II first. For the edge II, the Hamiltonian is decomposed as $H_{\text {eff }}=H_{m}+H_{p}$, with

$$
\begin{aligned}
H_{m}\left(k_{x},-i \partial_{y}\right) & =\left(E_{b}-B_{y} \partial_{y}^{2}\right) s_{0} \sigma_{z}+E_{d} s_{0} \sigma_{0}-i \alpha \partial_{y} s_{z} \sigma_{x}, \\
H_{p}\left(k_{x},-i \partial_{y}\right) & =-D_{y} \partial_{y}^{2} s_{0} \sigma_{0}-\eta k_{x} s_{0} \sigma_{y} .
\end{aligned}
$$

The purpose of this decomposition is to solve $H_{m}$ to obtain the two degenerate eigenfunctions of the edge states at $k_{x}=0$, then treat $H_{p}$ as the perturbation to obtain the linear dispersion terms. So the high-order $k_{x}^{2}$ terms have been omitted. We solve the equation $H_{m} \psi_{\alpha}(y)=E_{\alpha} \psi_{\alpha}(y)$ where $\alpha=$ 1,2 denote the two degenerate eigenstates at the same edge with the boundary condition $\psi_{\alpha}(0)=\psi_{\alpha}(\infty)=0$. The degenerate eigenenergy $E_{\alpha}=E_{d}$ and the eigenfunctions take the form

$$
\psi_{\alpha}(y)=\mathcal{N}_{y} \sin \left(\kappa_{1} y\right) e^{-\kappa_{2} y} e^{i k_{x} x} \tilde{\chi}_{\alpha}
$$

TABLE II. Fitting parameters of four bands model Hamiltonian.

\begin{tabular}{lccc}
\hline \hline$E_{d} / \mathrm{eV}$ & $E_{b} / \mathrm{eV}$ & $\alpha(\mathrm{eV} \AA)$ & $\eta(\mathrm{eV} \AA)$ \\
\hline 0.294 & 0.126 & 0.2 & 1.8 \\
$D_{x}\left(\mathrm{eV}^{2}\right)$ & $D_{y}\left(\mathrm{eV} \AA^{2}\right)$ & $B_{x}\left(\mathrm{eV} \AA^{2}\right)$ & $B_{y}\left(\mathrm{eV} \AA^{2}\right)$ \\
\hline 0.25 & 4.25 & -3.25 & -9.25 \\
\hline \hline
\end{tabular}



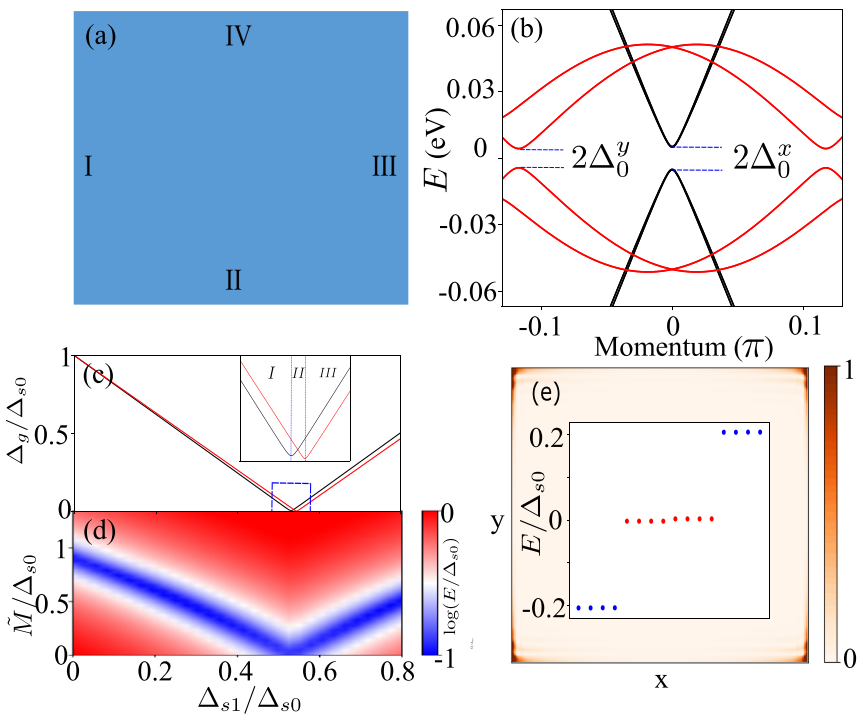

FIG. 5. (a) A rectangular with four edges which are labeled as I, II, III, and IV. (b) Band dispersions along $x$ (black) and $y$ (red) in Nambu space; (c) superconducting gap amplitudes at $x$ (black) and $y$ (red) edge $\Delta_{g}$ as a function of $\Delta_{s 1} / \Delta_{s 0}$. (d) The amplitude of minimal energy of $x$ edge (labeled by color) as a function of $\Delta_{s 1} / \Delta_{s 0}$ and magnetic field $\tilde{M}$. (e) The density plot of the MZMs with TR symmetry. The inset plot the several lowest eigenenergies.

with the normalization constant $\mathcal{N}_{y}=2 \sqrt{\kappa_{2}\left(\kappa_{1}^{2}+\kappa_{2}^{2}\right) / \kappa_{1}^{2}}$ and the two parameters $\kappa_{1}$ and $\kappa_{2}$ given by

$$
\kappa_{1}=\sqrt{-\frac{E_{b}}{B_{y}}-\frac{\alpha^{2}}{4 B_{y}^{2}}}, \quad \kappa_{2}=\frac{\alpha}{2 B_{y}} .
$$

$\tilde{\chi}_{\alpha}$ are eigenvectors satisfying $s_{z} \sigma_{y} \tilde{\chi}_{\alpha}=-\tilde{\chi}_{\alpha}$. Here we choose

$$
\tilde{\chi}_{1}=\left|\sigma_{y}=-1\right\rangle \otimes|\uparrow\rangle, \quad \tilde{\chi}_{2}=\left|\sigma_{y}=+1\right\rangle \otimes|\downarrow\rangle .
$$

The first- and the second-order perturbation Hamiltonian takes the form

$$
\begin{aligned}
& H_{\alpha, \alpha^{\prime}}^{(1)}=\left\langle\psi_{\alpha}\left|H_{p}\right| \psi_{\alpha^{\prime}}\right\rangle, \\
& H_{\alpha, \alpha^{\prime}}^{(2)}=\sum_{l} \frac{\left\langle\psi_{\alpha}\left|H_{p}\right| \psi_{l}\right\rangle\left\langle\psi_{l}\left|H_{p}\right| \psi_{\alpha^{\prime}}\right\rangle}{E_{\alpha}-E_{l}},
\end{aligned}
$$

where $\left|\psi_{l}\right\rangle$ denotes bulk states with energy $E_{l}$ and $\left|E_{\alpha}-E_{l}\right| \leqslant E_{b}$. According to Eqs.(9) and (10), we have

$$
H_{p}\left|\psi_{\alpha\left(\alpha^{\prime}\right)}\right\rangle=\left( \pm \eta k_{x}-\frac{D_{y} E_{b}}{B_{y}}\right)\left|\psi_{\alpha\left(\alpha^{\prime}\right)}\right\rangle+2 D_{y} \kappa_{1} \kappa_{2}\left|\phi_{\alpha\left(\alpha^{\prime}\right)}\right\rangle,
$$

where $\left|\phi_{\alpha}\right\rangle=\mathcal{N}_{y} \cos \left(\kappa_{1} y\right) e^{-\kappa_{2} y} e^{i k_{x} x} \tilde{\chi}_{\alpha}$, which is orthogonal to $\left|\psi_{\alpha}\right\rangle$. Therefore, the first and second terms on the right-hand side of Eq. (15) contribute to the first- and second-order perturbation as

$$
H^{(1)}=-\frac{D_{y} E_{b}}{B_{y}} \tilde{s}_{0}+\eta k_{x} \tilde{s}_{z}, H^{(2)} \approx-\frac{4\left(D_{y} \kappa_{1} \kappa_{2}\right)^{2}}{E_{b}} \tilde{s}_{0} .
$$

Here we choose Pauli matrices $\tilde{\boldsymbol{s}}$ to label edge states subspace. According to the Table II, we have $-\frac{D_{y} E_{b}}{B_{y}} \approx 0.058 \mathrm{eV}$ and
$-\frac{4\left(D_{y} \kappa_{1} \kappa_{2}\right)^{2}}{E_{b}} \approx-0.002 \mathrm{eV}$. This indicates that $H^{(1)}$ dominates the perturbation Hamiltonian and $H^{(2)}$ can be omitted. So the effective Hamiltonian of edge II takes the form

$$
H_{\mathrm{II}}\left(k_{x}\right)=\left(E_{d}-\frac{D_{y} E_{b}}{B_{y}}\right) \tilde{s}_{0}+\eta k_{x} \tilde{s}_{z} .
$$

By analogy, the effective Hamiltonian of the four edges are

$$
\begin{gathered}
H_{\mathrm{I}}\left(k_{y}\right)=M_{\mathrm{I}} \tilde{s}_{0}-\alpha k_{y} \tilde{s}_{z}, \quad H_{\mathrm{II}}\left(k_{x}\right)=M_{\mathrm{II}} \tilde{s}_{0}+\eta k_{x} \tilde{s}_{z}, \\
H_{\mathrm{III}}\left(k_{y}\right)=M_{\mathrm{III}} \tilde{s}_{0}+\alpha k_{y} \tilde{s}_{z}, \quad H_{\mathrm{IV}}\left(k_{x}\right)=M_{\mathrm{IV}} \tilde{s}_{0}-\eta k_{x} \tilde{s}_{z},
\end{gathered}
$$

where $M_{\mathrm{I}}=M_{\mathrm{III}}=E_{d}-\frac{D_{x} E_{b}}{B_{x}}, M_{\mathrm{II}}=M_{\mathrm{IV}}=E_{d}-\frac{D_{y} E_{b}}{B_{y}}$. This clearly shows that the energy difference of Dirac points $\delta E_{D}=$ $E_{b}\left(\frac{D_{x}}{B_{x}}-\frac{D_{y}}{B_{y}}\right)$ comes from the $C_{4}$ symmetry breaking. Taking into the fitting parameters (Table II), we find that $\delta E_{D}=$ $48 \mathrm{meV}$, which is consistent with numerical results of the eight bands model [Fig. 4(b)].

\section{LATTICE DISTORTION INDUCED SECOND-ORDER TPT}

To include the superconductivity, it is convenient to first write the electronic Hamiltonian in Nambu space as

$$
H_{\mathrm{n}}(k)=\left(H_{0}+H_{\mathrm{soc}}\right) \tau_{z},
$$

with $\tau_{z}$ the Pauli matrix acting on the particle-hole basis. In this case, each edge has two Dirac points near the Fermi level because the degree of freedom of the system is doubled. Note that as $C_{4}$ symmetry is broken by lattice distortion, the edge states should be considered separately for $x$ and $y$ edge. For simplicity, we consider the chemical potential $\mu=E_{u}$, that is, at the Dirac point of the $x$ edge [black curves in Fig. 4(b)]. When the superconducting gap is absent, the number of Dirac points at $k_{x}=0$ is doubled and the edge states are fourfold degenerates due to both time-reversal and charge $\mathrm{U}(1)$ symmetries. Along $y$ direction [red curves in Fig. 4(b)], the electronic Dirac point at $E_{l}$ is far below the chemical potential so that near the Fermi level there are two separated Dirac points, each of which is two-fold degenerate and protected solely by the charge U(1) symmetry.

\section{A. High-order TSC in DIII class}

The pairing symmetry of the monolayer FeSe still has many debates in various studies which give plain $s$-wave pairing [55-59], $s_{ \pm}$-wave pairing [60-64], and $d$-wave pairing [65-69]. Recent studies show that when the TI breaks $\mathrm{C}_{4}$ symmetry, both $s_{ \pm}$-wave pairing and $d$-wave pairing can lead to a pair of MZMs at each corner while plain $s$-wave pairing cannot $[38,40]$. Here, we do not intend to distinguish these pairing symmetries but show that the rectangular monolayer $\mathrm{FeSe}_{x} \mathrm{Te}_{1-x}$ can potentially be high-order time-reversal invariant TSC if it has $s_{ \pm}$-wave pairing. In this case, the Hamiltonian takes the form

$$
H_{\mathrm{sc}}(\boldsymbol{k})=H_{\mathrm{n}}(k)+\Delta(\boldsymbol{k}) \tau_{x},
$$

with $\Delta(\boldsymbol{k})=\Delta_{\mathrm{s} 0}-\Delta_{\mathrm{s} 1}\left(\cos \left(k_{x}\right)+\cos \left(k_{y}\right)\right)$. As the charge $\mathrm{U}(1)$ symmetries are broken so that the degeneracy at the 
Dirac points along $x$ and $y$ edges are lifted, which results in two gaps $\Delta_{0}^{x}$ and $\Delta_{0}^{y}$ [Fig. 5(b)]. By projecting the superconducting gap into the eigenfunctions of the edge state in Eq. (10), the effective gap of the two edges takes the form $\Delta_{0}^{x}=\left(2+\frac{E_{b}}{2 \bar{B}_{y}}\right) \Delta_{\mathrm{s} 1}-\Delta_{\mathrm{s} 0}$ and $\Delta_{0}^{y}=\left(2+\frac{E_{b}}{2 \bar{B}_{x}}\right) \Delta_{\mathrm{s} 1}-$ $\Delta_{\mathrm{s} 0}$ [40]. Since the break of $C_{4}$ symmetry makes $\tilde{B}_{y} \neq \tilde{B}_{x}$ where $\tilde{B}_{y}=\frac{B_{y}}{b^{2}}, \quad \tilde{B}_{x}=\frac{B_{x}}{a^{2}}, a$ and $b$ are lattice constants with the values of $B_{x}$ and $B_{y}$ given in Table II, MZMs can exist in the range

$$
\frac{1}{2+\frac{E_{b}}{2 \bar{B}_{y}}}<\frac{\Delta_{\mathrm{s} 1}}{\Delta_{\mathrm{s} 0}}<\frac{1}{2+\frac{E_{b}}{2 \tilde{B}_{x}}}
$$

[regime II in Fig. 5(c)], which is consistent with the results of Ref. [40]. In the regime of $\Delta_{0}^{x} \Delta_{0}^{y}<0$, each corner has a Kramers pair of MZMs [Fig. 5(e)]. The size of the topological regime is $1.5 \% \Delta_{\mathrm{s} 0}$ around $\Delta_{\mathrm{s} 1} / \Delta_{\mathrm{s} 0}=0.5$ for $\mathrm{s}_{ \pm}$-pairing symmetry according to our fitting parameters.

\section{B. High-order TSC in D class}

When applying a magnetic field induced Zeeman term $M s_{x}$ with $s$ the Pauli matrix acting on spin space, the time-reversal symmetry is broken and the gaps for $x$ and $y$ edges behave in very different manners. Without loss of generality, we firstly consider the edge in $x$ direction. Note that the $\mathrm{g}$ factor for the edge states maybe different from the bulk, we use $\tilde{M}$ to denote the effective Zeeman field strength through projecting $M s_{x}$ to the edge states (Appendix C). As the superconducting gap and the Zeeman term commute and the system has mirror x symmetry, $i s_{x}$, for $k_{x}=0$ and $\tilde{M}=0$, the four edge eigenstates can be characterized by the Pauli matrix $\tau_{x}$ and $s_{x}$ as $\left|\tau_{x}=1\right\rangle \otimes\left|s_{x}= \pm 1\right\rangle$ and $\left|\tau_{x}=-1\right\rangle \otimes\left|s_{x}= \pm 1\right\rangle$ with two gaps $\Delta_{1}^{x}=\Delta+\tilde{M}$ for $\tau_{x} \otimes s_{x}=1$ and $\Delta_{2}^{x}=|\Delta-\tilde{M}|$ for $\tau_{x} \otimes \sigma_{x}=-1$ [Fig 6(a)]. When $\tilde{M}$ is increased from $\tilde{M}<\Delta$ to $\tilde{M}>\Delta$, the gap of the edge states along the $x$ direction will undergo the closing and reopening at the transition point $\frac{\tilde{M}}{\Delta}=1$ [black curves in Fig. 6(b)]. This leads to a band inversion between $\left|\tau_{x}=-1\right\rangle \otimes\left|s_{x}=+1\right\rangle$ and $\left|\tau_{x}=1\right\rangle \otimes$ $\left|s_{x}=-1\right\rangle$ with the eigenvalues $\pm\left(\Delta_{0}^{x}-\tilde{M}\right)$. Meanwhile, the other two eigenstates with eigenvalues $\pm\left(\Delta_{0}^{x}+\tilde{M}\right)$ has no band inversion. Therefore, the eigenvalues of the superconducting matrix $\tau_{x}$ for the two eigenstates below Fermi level are changed from $v_{1}^{x}=v_{2}^{x}=-1$ to $v_{1}^{x}=-v_{2}^{x}=1$. Along $y$ direction, the gap amplitudes are almost independent of the Zeeman term [red curves in Fig. 6(b)] and have $\Delta_{1}^{y}=\Delta_{2}^{y} \approx$ $\Delta_{0}^{y}$ for $\tilde{M} \ll \delta E_{D}$ (Appendix C). We thus can define a $Z_{2}$ topological invariant $(-1)^{v}=\operatorname{sgn}\left(v_{1}^{x} v_{2}^{x}\right)$, which changes from 0 to 1 , indicating a second-order topological phase transition.

We further calculate the eigenvalues of the system for $v=0,1$. We found that for $v=1$ [Fig. 6(c)] there are four MZMs, localized at the four corners, which are absent for $v=0$. In Fig. 5(d), we show that there is always a Zeeman effect induced gap close, which is independent of the ratio $\Delta_{\mathrm{s} 1} / \Delta_{\mathrm{s} 0}$. This means the implementation of Majorana corner state in D class monolayer $\mathrm{FeSe}_{x} \mathrm{Te}_{1-x}$ is not sensitive to the superconducting pairing symmetries. Without loss of generality, we take $\Delta_{\mathrm{s} 1}=0$ in the rest of this paper. So far, the chemical potential is taken $\mu=E_{u}$. We further calculate the lowest eigenenergy of the closed system as a function of
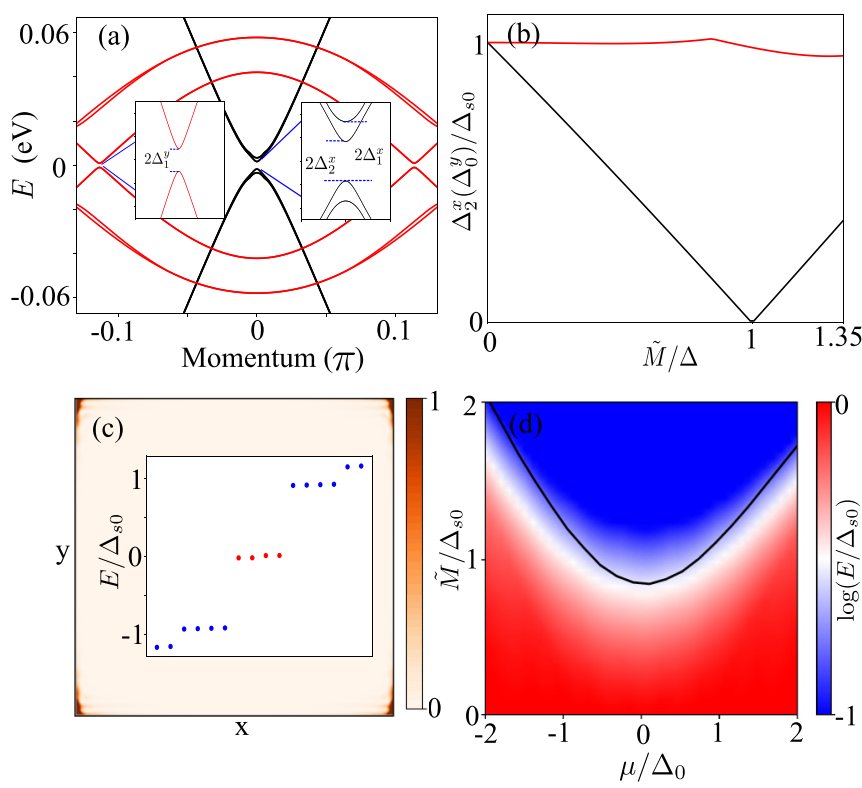

FIG. 6. (a) Band dispersions along $x$ (black) and $y$ (red) in Nambu space with a magnetic field. (b) The gap of the edge states along the $x$ (black) and $y$ (red) direction and as a function of magnetic field. (c) The density plot of the MZMs with a magnetic field, which breaks TR symmetry. The inset plot the several lowest eigenenergies. (d) Phase diagram with chemical potential and magnetic field. The blue color indicates the existence of Majorana corner states.

chemical potential and magnetic field $M$ [Fig. 6(d)]. The color plot of the eigenenergy shows an obvious phase boundary between zero (blue) and finite (red) values. We also calculate the critical magnetic field [black curve in Fig. 6(d)], where the gap of the edge state along $x$ direction is closed, as a function of chemical potential. The phase boundary matches the critical magnetic field well. This means the Majorana corner states of D class in our work are not sensitive to the chemical potential as long as it does not close the edge states gap. It is noted that the protection of the Majorana Kramers pairs requires timereversal symmetry, and thus their braiding operation has some symmetry restrictions [70]. While the single MZM at each corner is protected only by particle-hole symmetry, therefore their braiding operation has no symmetry restriction and is more robust. The topological invariant $v$ defined through the edge state Hamiltonian is consistent with the Pfaffian calculation when the system takes the periodic boundary condition along $x$ direction and the open boundary condition along $y$ direction. The magnetic field is only applied to the lower half-plane [Fig. 7(a)]. The system can be considered as quasi1D with many transverse modes with Hamiltonian taking the form $H_{s c}\left(k_{x}, y\right)$. The topology of this superconducting slab geometry can be characterized by the topological invariant $v_{0}=\operatorname{sgn}\left(\frac{\operatorname{Pf}\left[\mathrm{H}\left(\mathrm{k}_{\mathrm{x}}=0, \mathrm{y}\right)\right]}{\operatorname{Pf}\left[\mathrm{H}\left(\mathrm{k}_{\mathrm{x}}=\pi, \mathrm{y}\right)\right]}\right)$ with \pm 1 correspond to the even and odd numbers of $1 \mathrm{D}$ topological superconducting chain in the system. We then plot $v_{0}$ as a function of the Zeeman splitting energy $\tilde{M}$ in Fig. 7(b) which shows a sudden drop from 1 to -1 at the edge state gap closing point $\frac{\tilde{M}}{\Delta}=1$ and characters the phase transition. Both $v$ and $v_{0}$ changes when $\frac{\tilde{M}}{\Delta}=1$, so these two topological invariants are equivalent. As the system 

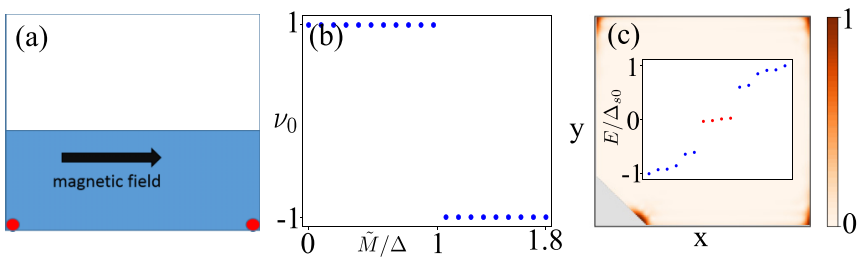

FIG. 7. (a) A slab with magnetic field applied in the half of the system (blue region). The red points indicate the existence of the Majorana zero modes. (b) Topological invariant $v_{0}$ as a function of the magnetic field. (c) The density distribution and energy of MZMs when the shape of the corner is not perfect.

with the magnetic field along $x$ direction has mirror symmetry, we can also define the edge state Wannier function, whose center is quantized 0 or $1 / 2$ due to the mirror symmetry. In the Appendix D, we show the change of the quantization of the edge state Wannier center also happens at $\frac{\tilde{M}}{\Delta}=1$.

\section{DISCUSSION AND CONCLUSION}

Considering the experimental reality, the $d$ orbitals of the iron-based superconductors may have a very complicated $g$ factor, resulting in orbital dependent Zeeman term other than $M \sigma_{0} s_{x}$. However, the various Zeeman terms can always lead to the transition from $v=0$ to $v=1$, while the details are given in Appendix F. Therefore, our results are independent of the special Zeeman forms. The edges of the iron-based superconductor may not be perfectly along $x$ or $y$ directions, and the corner maybe not sharp, which, however, does not affect the robustness of the Majorana corner state due to its protection only from particle-hole symmetry. In Fig. 7(c), the four eigenfunctions with the lowest eigenvalues are plotted in the system whose two neighbor edges have an angle of $105^{\circ}$ with smooth corners. These states localize at the four corners with perfect zero energy, which indicates the robustness of the MZMs under the imperfect edges and corners. In conclusion, the $\mathrm{C}_{4}$ symmetry breaking by the lattice distortion in monolayer $\mathrm{FeSe}_{x} \mathrm{Te}_{1-x}$ can lead to the first-order topological phase transition in the wide composition range $x \in(0,0.7)$,which has a large overlap with the composition range of the high- $T_{c}$ monolayer $\mathrm{FeSe}_{x} \mathrm{Te}_{1-x}$ [53,71-73]. This rectangular monolayer $\mathrm{FeSe}_{x} \mathrm{Te}_{1-x}$ is also a promising candidate to realize Majorana corner states.

\section{ACKNOWLEDGMENTS}

We would like to thank Chao-Xing Liu, Chen Fang, Ling-Yuan Kong, and Yi Zhou for fruitful discussions. G.X. acknowledges the support of the Ministry of Science and Technology of China (Grant No. 2018YFA0307000), and the National Natural Science Foundation of China (Grant No. 11874022). X.L. acknowledges the Ministry of Science and Technology of China (Grant No. 2016YFA0401003) and the National Natural Science Foundation of China (Grants No. 11674114 and No. 12074133). (a)

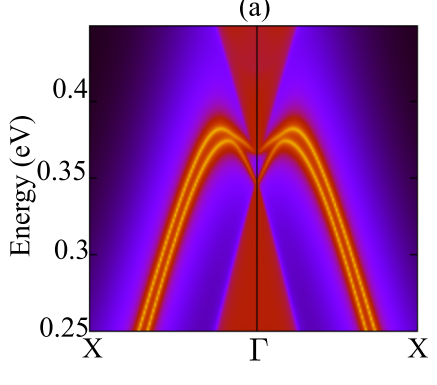

(c)

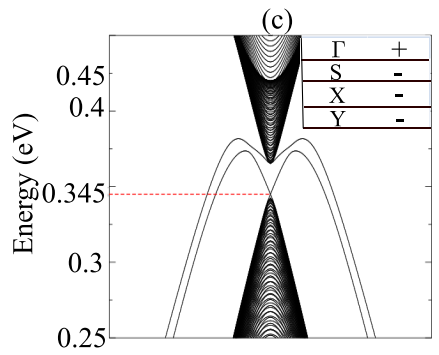

(b)

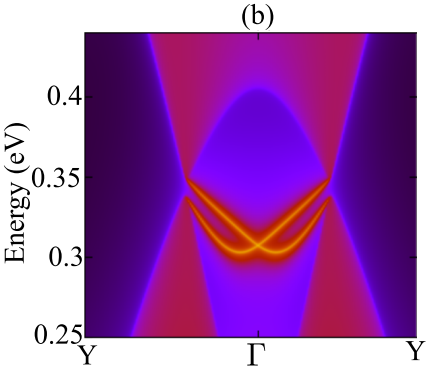

(d)

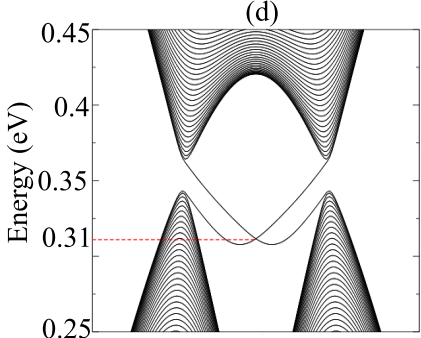

FIG. 8. (a) Energy and momentum dependence of LDOS for monolayer $\mathrm{FeSe}(\mathrm{Te})$ on the (100) edge. The higher LDOS is represented by brighter color. (b) LDOS for monolayer $\mathrm{FeSe}(\mathrm{Te})$ on the (010) edge. (c) Energy spectrum for (100) edge. (d) Energy spectrum for (010) edge.

\section{APPENDIX A: DFT CALCULATIONS METHOD}

Our first-principles calculations are implemented in the framework of generalized gradient approximation with the Perdew-Burke-Ernzerhof functional using the Vienna ab initio simulation package (VASP) [74]. The lattice constant $a=$ $3.6707 \AA, b=3.9050 \AA$, and the heights of $\mathrm{Se} / \mathrm{Te}$ are relaxed among all the calculations, and all the self-consistent calculations are carried out on $15 \times 15 \times 2 k$-point mesh. The cutoff energy (ENCUT) of the wave function is set to be $268 \mathrm{eV}$ in our cases. To ensure the decoupling between neighboring slabs, we set the vacuum layer to be $15 \AA$. Besides, we adopt virtual crystal approximation to make the substitution proper. We also perform a series of SOC band calculations with different exchange-correlation functions such as LDA, PW91, and PBE and obtain a similar energy gap, which indicates that our result is independent of the exchange-correlation functions. Our previous calculations of the topological band gap in 3D bulk $\mathrm{Fe}(\mathrm{Se}, \mathrm{Te})$ with the same methods [20] give a similar energy scale, which has been confirmed by other theoretical calculations $[14,73]$ and experiments $[17,21]$.

\section{APPENDIX B: EDGES STATES OBTAINED FROM DFT CALCULATIONS}

Maximally localized Wannier functions (MLWFs) [75] for the $3 d$ orbitals of $\mathrm{Fe}$ and the $4 p$ orbitals of Se are generated from monolayer calculations. We calculate the edge states iteratively by constructing the tight-binding (TB) Hamiltonians of semi-infinite sample [76,77]. Besides, iterative Green's function is necessary for obtaining the surface state spectrum, and we use the open-source software package WANNIERTOOLS [78] to fulfill our work. The (100) and (010) edge local density of states (LDOS) of $\mathrm{FeSe}(\mathrm{Te})$ are shown in Figs. 8(a) and 8 (b), respectively. To see it more clearly, we also calculate the 


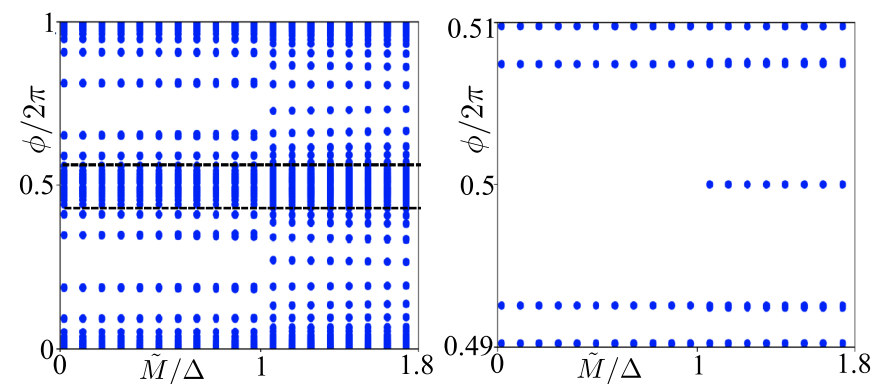

FIG. 9. Left panel: Wilson loop as a function of the magnetic field. Right panel: the inset for the region of the black dash lines.

spectrums of the two edges [Figs. 8(c) and 8(d)]. The Dirac points of the two edges are shown in the red dash lines. The energy difference of the two Dirac points $\delta E_{D}$ is $35 \mathrm{meV}$, which is slightly different from that in the main text. However, this will not affect our results because $\delta E_{D}$ is still very large compared with the superconductivity and the applied magnetic field. As shown in Fig. 8(c), the Dirac point is not very clear as it is very close to the bulk states. To verify its existence, we make a parity analysis, given in the inset of Fig. 8(c). The difference of the parity between $\Gamma$ point and the other three time-reversal invariant points confirm the existence of the Dirac point at $\Gamma$ point for (100) edge.

\section{APPENDIX C: EFFECTIVE GAP OF EDGES WITH MAGNETIC FIELD AND SUPERCONDUCTIVITY}

In this section, we consider how the magnetic field and superconductivity affect the gap of the two perpendicular edges [for example, edge II and edge III in Fig. 5(a)]. If we tune the chemical potential near the Dirac point of edge II, say $\mu=M_{I I}$, according to Eq.(18), the BdG Hamiltonian of edge II and III with superconductivity and magnetic field takes the form

$$
\begin{aligned}
& H_{B d G}^{I I}=\eta k_{x} \tau_{z} \tilde{s}_{z}+\Delta \tau_{x} \tilde{s}_{0}+\tilde{M} \tau_{0} \tilde{s}_{x}, \\
& H_{B d G}^{I I I}=\delta E_{D} \tau_{z} \tilde{s}_{0}+\alpha k_{y} \tau_{z} \tilde{s}_{z}+\Delta \tau_{x} \tilde{s}_{0}+\tilde{M} \tau_{0} \tilde{s}_{x},
\end{aligned}
$$

where $\tilde{M}$ and $\Delta$ are the magnetic field and pairing strength. Then we can obtain the eigenvalues by diagonalizing the Hamiltonian of the two edges. For edge II, we have

$$
E_{I I}= \pm \sqrt{\Delta^{2}+\eta^{2} k_{x}^{2} \pm 2 \Delta \tilde{M}}
$$

The gap of the edge II is determined by the relation $|\tilde{M}-\Delta|$. While for edge III, the gap is given by $\sqrt{\Delta^{2}-2\left(\delta E_{D}\right)^{2}\left(\sqrt{1+\frac{\Delta^{2} \tilde{M}^{2}}{\left(\delta E_{D}\right)^{4}}+\frac{\tilde{M}^{2}}{\left(\delta E_{D}\right)^{2}}}-1\right)+\tilde{M}^{2}}$. In our cases, $\delta E_{D} \gg \Delta, \tilde{M}$, so the gap $\approx \Delta$. This indicates that the second-order phase transition of the system is only determined by the edge in (100) direction, as discussed in the main text. In the eight-bands model, the magnetic filed term $M s_{x}$ is not equivalent to $\tilde{M} \tilde{s}_{x}$ in Eq. (C1). The latter refers to the Zeeman splitting of edge states, which can be obtained from the projection. In our cases, we have $M \approx 3 \tilde{M}$.

\section{APPENDIX D: CALCULATION OF WANNIER CENTER}

Note that our model Hamiltonian in Eq. (20) of the main text has mirror symmetry. The applied magnetic field along $x$ direction preserves the mirror symmetry for $x$ direction. This can reflect in the Wilson loop calculations in the slab geometry (Fig. 9), which gives the Wannier wave function center of Majorana modes, referred to as Majorana Wannier center. Now we consider the system takes periodic along the $x$ direction and the open boundary condition along the $y$ direction. So according to the Hamiltonian $H_{s c}\left(k_{x}, y\right)$, we calculate the logarithm of the Wilson loop of the negative energy band as a function of the Zeeman splitting $\tilde{M}$ with fixing superconductivity $\Delta$, as shown in Fig. 9. The inset shows a sudden appearance of $\pm 1 / 2$ when Zeeman splitting reaches a critical value $\tilde{M}=\Delta$, which is the transition point of the topological invariant $v_{0}$. The Wilson loop in Fig. 9 distributes symmetrically around $1 / 2$ because our Hamiltonian has mirror symmetry. However, Majorana corner states in our system still exist when the Mirror symmetry is weakly broken [Fig. 7(c) in the main text], because it is also protected by particle-hole symmetry.

\section{APPENDIX E: PARAMETERS USED TO CALCULATE MAJORANA CORNER STATES}

As discussed in the main text, we use the different parameters from that in Table I for the calculation of Fig. 5(e), Figs. 6(c), 6(d), and Figs. 7(b)-7(d). These figures reveal the existence of the Majorana corner states, so we need to calculate the eigenvalues of the system with a finite size. However, if we use the parameters in Table I, it is impossible to obtain the Majorana corner states with nearly zero energies because of the calculation ability. To decrease the coherent length of the system, we use the parameters shown in Table III. Although the parameters are not realistic, we emphasize that it will not affect our results. We calculate the edge states by using the parameters in Table III, as shown in Fig. 10(a). The

TABLE III. Parameters for calculations of Majorana corner states in class D and DIII. For class DIII, only the adjusted parameters are listed in the bracket, while the other unlisted parameters are the same as those in class D.

\begin{tabular}{lccccccccc}
\hline \hline \multicolumn{7}{c}{ class D (class DIII) } \\
\hline$E_{1} / \mathrm{eV}$ & $E_{2} / \mathrm{eV}$ & $E_{3} / \mathrm{eV}$ & $E_{4} / \mathrm{eV}$ & $\beta\left(\mathrm{eV} \AA^{2}\right)$ & $M_{1 x}\left(\mathrm{eV} \AA^{2}\right)$ & $M_{1 y}\left(\mathrm{eV} \AA^{2}\right)$ & $M_{2 x}\left(\mathrm{eV} \AA^{2}\right)$ & $M_{2 y}\left(\mathrm{eV} \AA^{2}\right)$ \\
\hline 0.07 & 0.2 & $0.19(0.45)$ & 0.15 & 2.44 & -1.75 & -3.51 & -2.02 & -1.07 \\
$\lambda_{1} / \mathrm{eV}$ & $\lambda_{2} / \mathrm{eV}$ & $\lambda_{3} / \mathrm{eV}$ & $\delta(\mathrm{eV} \AA)$ & $\gamma(\mathrm{eV} \AA)$ & $M_{3 x}\left(\mathrm{eV} \AA^{2}\right)$ & $M_{3 y}\left(\mathrm{eV} \AA^{2}\right)$ & $M_{4 x}\left(\mathrm{eV} \AA^{2}\right)$ & $M_{4 y}\left(\mathrm{eV} \AA^{2}\right)$ \\
\hline 0.52 & 0.35 & 0.45 & 1.87 & 1.02 & $-2.96(-1.61)$ & $-0.91(-3.94)$ & 3.10 & $4.88(9.46)$ \\
\hline \hline
\end{tabular}


(a)
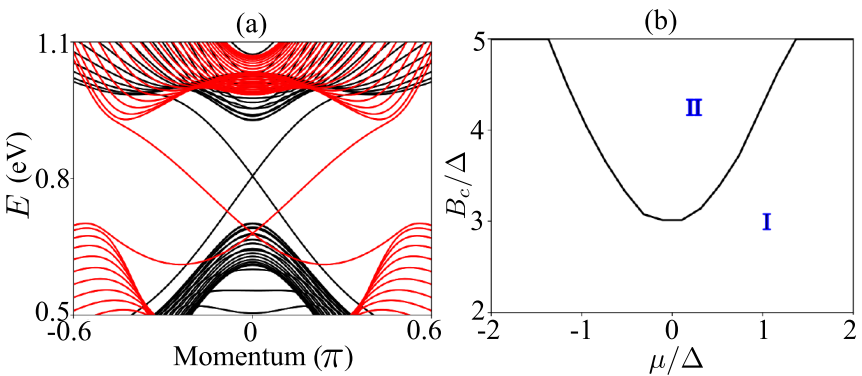

FIG. 10. (a) Edge states in (100) (black lines) and (010)(red lines) directions obtained from the parameters in Table III. (b) Phase diagram obtained from the realistic parameters (Table I).

properties of the two edges are qualitatively consistent with those obtained from the realistic parameters.

As shown in Fig. 6(d) in the main text, Majorana corner states will exist as long as the magnetic field is larger than the critical value, which does not depend on the specific parameters used in the calculation. With this consideration, we calculate the critical magnetic field $B_{c}$, where the gap of the edge state along $x$ direction is closed, as a function of chemical potential with the realistic parameters (Table I) and obtain the phase diagram as shown in Fig. 10(b). The critical temperature of the monolayer $\mathrm{FeSe}(\mathrm{Te})$ is around $10-15 \mathrm{meV}$ [53], while the proximity-induced superconducting gap at the edges is normally one order smaller. So we choose superconducting gap $\Delta$ to be $1 \mathrm{meV}$ and find that minimal critical value of the magnetic energy relating to $3 \mathrm{meV}$, corresponding to a magnetic field of $26 \mathrm{~T}$ assuming the $\mathrm{g}$ factor $g_{0}=2$. Fortunately, this magnetic field is well below the in-plane critical magnetic field of monolayer $\mathrm{FeSe}(\mathrm{Te})$, which is about $45 \mathrm{~T}[79]$.

\section{APPENDIX F: EFFECT OF THE MAGNETIC FIELD TERMS}

In the main text, all the calculations are performed with the magnetic field in (100) direction. Here we will show that our results do not depend on the specific direction of the in-plane magnetic field. At first, we calculate the critical magnetic field
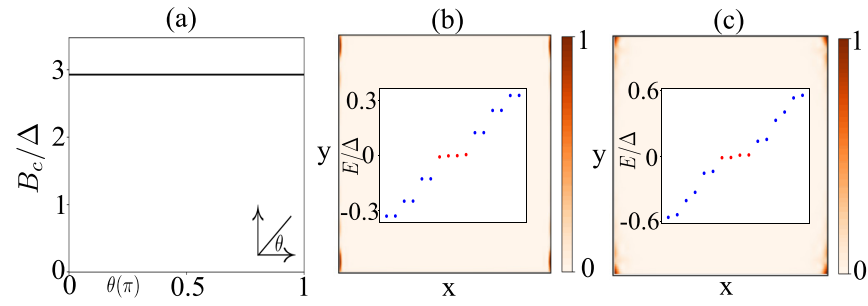

FIG. 11. (a) The critical magnetic field $B_{c}$ as a function of the direction of the in-plane magnetic field. (b) Majorana corner states with magnetic field along $y$ direction $\left(\theta=90^{\circ}\right)$. (c) Majorana corner states for the Zeeman term including both the spin and angular momentums couplings as shown in Eq. (F1).

$B_{c}$, where the gap of the edge state along $x$ direction is closed, as a function of the angle $\theta$ of the in-plane magnetic field, as shown in Fig. 11(a). The critical value $B_{c}$ hardly depends on $\theta$. Then we calculate the eigenvalues of the system with $\theta=90^{\circ}$ [Fig. 11(b)] and find that four Majorana corner states exist at the corners, which indicates that our results do not rely on the direction of the magnetic field. The angular momentums of the states may also affect the Zeeman coupling. Then the Zeeman terms of the Hamiltonian takes the form [48]

$$
H_{z}=\mu_{B}\left(g_{s} \boldsymbol{S}+g_{l} \boldsymbol{L}\right) \cdot \boldsymbol{B} .
$$

In the basis $\left(|y z\rangle,\left|x^{2}-y^{2}\right\rangle,|x z\rangle,|z\rangle\right), L_{x}, L_{y}$, and $L_{z}$ are given by

$$
\begin{aligned}
L_{x} & =\left(\begin{array}{cccc}
0 & -i & 0 & 0 \\
-i & 0 & 0 & 0 \\
0 & 0 & 0 & 0 \\
0 & 0 & 0 & 0
\end{array}\right), \quad L_{y}=\left(\begin{array}{cccc}
0 & 0 & 0 & 0 \\
0 & 0 & i & 0 \\
0 & -i & 0 & 0 \\
0 & 0 & 0 & 0
\end{array}\right) \\
L_{z} & =\left(\begin{array}{cccc}
0 & 0 & i & 0 \\
0 & 0 & 0 & 0 \\
-i & 0 & 0 & 0 \\
0 & 0 & 0 & 0
\end{array}\right) .
\end{aligned}
$$

We calculate the eigenvalues of the system with Zeeman terms in Eq. (F1), as shown in Fig. 11(a). This leads the Majorana corner states can exist independently with the in-plane magnetic field direction [Fig. 11(b)] and the specific Zeeman terms [Fig. 11(c)].
[1] L. Fu and C. L. Kane, Superconducting Proximity Effect and Majorana Fermions at the Surface of a Topological Insulator, Phys. Rev. Lett. 100, 096407 (2008).

[2] R. M. Lutchyn, J. D. Sau, and S. Das Sarma, Majorana Fermions and a Topological Phase Transition in Semiconductor-Superconductor Heterostructures, Phys. Rev. Lett. 105, 077001 (2010).

[3] Y. Oreg, G. Refael, and F. von Oppen, Generic New Platform for Topological Quantum Computation Using Semiconductor Heterostructures, Phys. Rev. Lett. 105, 177002 (2010).

[4] J. D. Sau, R. M. Lutchyn, S. Tewari, and S. Das Sarma, Generic New Platform for Topological Quantum Computation Using Semiconductor Heterostructures, Phys. Rev. Lett. 104, 040502 (2010).
[5] J. Alicea, Majorana fermions in a tunable semiconductor device, Phys. Rev. B 81, 125318 (2010).

[6] J.-P. Xu, C. Liu, M.-X. Wang, J. Ge, Z.-L. Liu, X. Yang, Y. Chen, Y. Liu, Z.-A. Xu, C.-L. Gao, D. Qian, F.-C. Zhang, and J.-F. Jia, Artificial Topological Superconductor by the Proximity Effect, Phys. Rev. Lett. 112, 217001 (2014).

[7] H. Zhao, B. Rachmilowitz, Z. Ren, R. Han, J. Schneeloch, R. Zhong, G. Gu, Z. Wang, and I. Zeljkovic, Superconducting proximity effect in a topological insulator using $\mathrm{Fe}(\mathrm{Te}, \mathrm{Se})$, Phys. Rev. B 97, 224504 (2018).

[8] F.-C. Hsu, J.-Y. Luo, K.-W. Yeh, T.-K. Chen, T.-W. Huang, P. M Wu, Y.-C. Lee, Y.-L. Huang, Y.-Y. Chu, D.-C. Yan, and M.-K. $\mathrm{Wu}$, Superconductivity in the pbo-type structure $\alpha$-FeSe, Proc. Natl. Acad. Sci. USA 105, 14262 (2008). 
[9] T. Hanaguri, S. Niitaka, K. Kuroki, and H. Takagi, Unconventional $s$-wave superconductivity in $\mathrm{Fe}(\mathrm{Se}, \mathrm{Te})$, Science 328, 474 (2010).

[10] C.-L. Song, Y.-L. Wang, P. Cheng, Y.-P. Jiang, W. Li, T. Zhang, Z. Li, K. He, L. Wang, J.-F. Jia, H.-H. Hung, C. Wu, X. Ma, X. Chen, and Q.-K. Xue, Direct observation of nodes and twofold symmetry in FeSe superconductor, Science 332, 1410 (2011).

[11] Q.-Y. Wang, Z. Li, W.-H. Zhang, Z.-C. Zhang, J.-S. Zhang, W. Li, H. Ding, Y.-B. Ou, P. Deng, K. Chang, J. Wen, C.-L. Song, K. He, J.-F. Jia, S.-H. Ji, Y.-Y. Wang, L.-L. Wang, X. Chen, X.-C. Ma, and Q.-K. Xue, Interface-induced high-temperature superconductivity in single unit-cell FeSe films on $\mathrm{SrTiO}_{3}$, Chin. Phys. Lett. 29, 037402 (2012).

[12] J.-F. Ge, Z.-L. Liu, C. Liu, C.-L. Gao, D. Qian, Q.-K. Xue, Y. Liu, and J.-F. Jia, Superconductivity above $100 \mathrm{~K}$ in single-layer FeSe films on doped $\mathrm{SrTiO}_{3}$, Nat. Mater. 14, 285 (2014).

[13] F. Li, H. Ding, C. Tang, J. Peng, Q. Zhang, W. Zhang, G. Zhou, D. Zhang, C.-L. Song, K. He, S. Ji, X. Chen, L. Gu, L. Wang, X.-C. Ma, and Q.-K. Xue, Interface-enhanced hightemperature superconductivity in single-unit-cell $\mathrm{FeTe}_{1-x} \mathrm{Se}_{x}$ films on $\mathrm{SrTiO}_{3}$, Phys. Rev. B 91, 220503(R) (2015).

[14] Z. Wang, P. Zhang, G. Xu, L. K. Zeng, H. Miao, X. Xu, T. Qian, H. Weng, P. Richard, A. V. Fedorov, H. Ding, X. Dai, and Z. Fang, Topological nature of the $\mathrm{FeSe}_{0.5} \mathrm{Te}_{0.5}$ superconductor, Phys. Rev. B 92, 115119 (2015).

[15] X. Wu, S. Qin, Y. Liang, H. Fan, and J. Hu, Topological characters in $\mathrm{Fe}\left(\mathrm{Te}_{1-x} \mathrm{Se}_{x}\right)$ thin films, Phys. Rev. B 93, 115129 (2016).

[16] P. Zhang, K. Yaji, T. Hashimoto, Y. Ota, T. Kondo, K. Okazaki, Z. Wang, J. Wen, G. D. Gu, H. Ding, and S. Shin, Observation of topological superconductivity on the surface of an iron-based superconductor, Science 360, 182 (2018).

[17] P. Zhang, Z. Wang, X. Wu, K. Yaji, Y. Ishida, Y. Kohama, G. Dai, Y. Sun, C. Bareille, K. Kuroda, T. Kondo, K. Okazaki, K. Kindo, X. Wang, C. Jin, J. Hu, R. Thomale, K. Sumida, S. Wu, $\mathrm{K}$. Miyamoto et al., Multiple topological states in iron-based superconductors, Nat. Phys. 15, 41 (2019).

[18] X.-L. Peng, Y. Li, X.-X. Wu, H.-B. Deng, X. Shi, W.-H. Fan, M. Li, Y.-B. Huang, T. Qian, P. Richard, J.-P. Hu, S.-H. Pan, H.-Q. Mao, Y.-J. Sun, and H. Ding, Observation of topological transition in high- $\mathrm{T}_{c}$ superconductor $\mathrm{FeTe}_{1-x} \mathrm{Se}_{x} / \mathrm{SrTiO}_{3}(001)$ monolayers, Phys. Rev. B 100, 155134 (2019).

[19] P. Hosur, P. Ghaemi, R. S. K. Mong, and A. Vishwanath, Majorana Modes at the Ends of Superconductor Vortices in Doped Topological Insulators, Phys. Rev. Lett. 107, 097001 (2011).

[20] G. Xu, B. Lian, P. Tang, X.-L. Qi, and S.-C. Zhang, Topological Superconductivity on the Surface of Fe-Based Superconductors, Phys. Rev. Lett. 117, 047001 (2016).

[21] Q. Liu, C. Chen, T. Zhang, R. Peng, Y.-J. Yan, C.-H.-P. Wen, X. Lou, Y.-L. Huang, J.-P. Tian, X.-L. Dong, G.-W. Wang, W.-C. Bao, Q.-H. Wang, Z.-P. Yin, Z.-X. Zhao, and D.-L. Feng, Robust and Clean Majorana Zero Mode in the Vortex Core of High-Temperature Superconductor $\left(\mathrm{Li}_{0.84} \mathrm{Fe}_{0.16}\right) \mathrm{OHFeSe}$, Phys. Rev. X 8, 041056 (2018).

[22] L. Kong, S. Zhu, M. Papaj, H. Chen, L. Cao, H. Isobe, Y. Xing, W. Liu, D. Wang, P. Fan, Y. Sun, S. Du, J. Schneeloch, R. Zhong, G. Gu, L. Fu, H.-J. Gao, and H. Ding, Observation of half-integer level shift of vortex bound states in an iron-based superconductor, Nat. Phys. 15, 1181 (2019).
[23] S. Zhu, L. Kong, L. Cao, H. Chen, M. Papaj, S. Du, Y. Xing, W. Liu, D. Wang, C. Shen, F. Yang, J. Schneeloch, R. Zhong, G. Gu, L. Fu, Y.-Y. Zhang, H. Ding, and H.-J. Gao, Observation of Majorana conductance plateau by scanning tunneling spectroscopy, Science 367, 189 (2020).

[24] F. Zhang, C. L. Kane, and E. J. Mele, Surface State Magnetization and Chiral Edge States on Topological Insulators, Phys. Rev. Lett. 110, 046404 (2013).

[25] W. A. Benalcazar, B. A. Bernevig, and T. L. Hughes, Electric multipole moments, topological multipole moment pumping, and chiral hinge states in crystalline insulators, Phys. Rev. B 96, 245115 (2017).

[26] J. Langbehn, Y. Peng, L. Trifunovic, F. von Oppen, and P. W. Brouwer, Reflection-Symmetric Second-Order Topological Insulators and Superconductors, Phys. Rev. Lett. 119, 246401 (2017).

[27] Z. Song, Z. Fang, and C. Fang, $(d-2)$-Dimensional Edge States of Rotation Symmetry Protected Topological States, Phys. Rev. Lett. 119, 246402 (2017).

[28] M. Ezawa, Higher-Order Topological Insulators and Semimetals on the Breathing Kagome and Pyrochlore Lattices, Phys. Rev. Lett. 120, 026801 (2018).

[29] H. Shapourian, Y. Wang, and S. Ryu, Topological crystalline superconductivity and second-order topological superconductivity in nodal-loop materials, Phys. Rev. B 97, 094508 (2018).

[30] X. Zhu, Tunable majorana corner states in a two-dimensional second-order topological superconductor induced by magnetic fields, Phys. Rev. B 97, 205134 (2018).

[31] M. Geier, L. Trifunovic, M. Hoskam, and P. W. Brouwer, Second-order topological insulators and superconductors with an order-two crystalline symmetry, Phys. Rev. B 97, 205135 (2018).

[32] E. Khalaf, Higher-order topological insulators and superconductors protected by inversion symmetry, Phys. Rev. B 97, 205136 (2018).

[33] F. Schindler, A. M. Cook, M. G. Vergniory, Z. Wang, S. S. P. Parkin, B. A. Bernevig, and T. Neupert, Higher-order topological insulators, Sci. Adv. 4, eaat0346 (2018).

[34] M. Ezawa, Strong and weak second-order topological insulators with hexagonal symmetry and $\mathbb{Z}_{3}$ index, Phys. Rev. B 97, 241402(R) (2018).

[35] F. Schindler, Z. Wang, M. G. Vergniory, A. M. Cook, A. Murani, S. Sengupta, A. Y. Kasumov, R. Deblock, S. Jeon, I. Drozdov, H. Bouchiat, S. Guéron, A. Yazdani, B. A. Bernevig, and T. Neupert, Higher-order topology in bismuth, Nat. Phys. 14, 918 (2018).

[36] M. Ezawa, Minimal models for Wannier-type higher-order topological insulators and phosphorene, Phys. Rev. B 98, 045125 (2018).

[37] M. Ezawa, Magnetic second-order topological insulators and semimetals, Phys. Rev. B 97, 155305 (2018).

[38] Q. Wang, C.-C. Liu, Y.-M. Lu, and F. Zhang, High-Temperature Majorana Corner States, Phys. Rev. Lett. 121, 186801 (2018).

[39] V. Dwivedi, C. Hickey, T. Eschmann, and S. Trebst, Majorana corner modes in a second-order Kitaev spin liquid, Phys. Rev. B 98, 054432 (2018).

[40] Z. Yan, F. Song, and Z. Wang, Majorana Corner Modes in a High-Temperature Platform, Phys. Rev. Lett. 121, 096803 (2018). 
[41] Y. Wang, M. Lin, and T. L. Hughes, Weak-pairing higher order topological superconductors, Phys. Rev. B 98, 165144 (2018).

[42] C.-H. Hsu, P. Stano, J. Klinovaja, and D. Loss, Majorana Kramers Pairs in Higher-Order Topological Insulators, Phys. Rev. Lett. 121, 196801 (2018).

[43] X.-H. Pan, K.-J. Yang, L. Chen, G. Xu, C.-X. Liu, and X. Liu, Lattice Symmetry Assisted Second Order Topological Superconductors and Majorana Patterns, Phys. Rev. Lett. 123, 156801 (2019).

[44] R.-X. Zhang, W. S. Cole, and S. Das Sarma, Helical Hinge Majorana Modes in Iron-Based Superconductors, Phys. Rev. Lett. 122, 187001 (2019).

[45] R.-X. Zhang, W. S. Cole, X. Wu, and S. Das Sarma, HigherOrder Topology and Nodal Topological Superconductivity in $\mathrm{Fe}(\mathrm{Se}, \mathrm{Te})$ Heterostructures, Phys. Rev. Lett. 123, 167001 (2019).

[46] Y.-T. Hsu, W. S. Cole, R.-X. Zhang, and J. D. Sau, Inversion-Protected Higher-Order Topological Superconductivity in Monolayer $\mathrm{WTe}_{2}$, Phys. Rev. Lett. 125, 097001 (2020).

[47] S.-B. Zhang and B. Trauzettel, Detection of second-order topological superconductors by Josephson junctions, Phys. Rev. Research 2, 012018(R) (2020).

[48] X. Wu, X. Liu, R. Thomale, and C.-X. Liu, High- $T_{c}$ superconductor $\mathrm{Fe}(\mathrm{Se}, \mathrm{Te})$ monolayer: An intrinsic, scalable, and electrically-tunable Majorana platform, Natl. Sci. Rev., nwab087 (2021).

[49] P. Zhang, X.-L. Peng, T. Qian, P. Richard, X. Shi, J.-Z. Ma, B. B. Fu, Y.-L. Guo, Z. Q. Han, S. C. Wang, L. L. Wang, Q.-K. Xue, J. P. Hu, Y.-J. Sun, and H. Ding, Observation of high- $T_{c}$ superconductivity in rectangular $\mathrm{FeSe} / \mathrm{SrTiO}_{3}(110)$ monolayers, Phys. Rev. B 94, 104510 (2016).

[50] P. Hohenberg and W. Kohn, Inhomogeneous electron gas, Phys. Rev. 136, B864 (1964).

[51] W. Kohn and L. J. Sham, Self-consistent equations including exchange and correlation effects, Phys. Rev. 140, A1133 (1965).

[52] K. Liu, Z.-Y. Lu, and T. Xiang, Atomic and electronic structures of fese monolayer and bilayer thin films on $\mathrm{SrTiO}_{3}(001)$ : Firstprinciples study, Phys. Rev. B 85, 235123 (2012).

[53] X. Shi, Z.-Q. Han, P. Richard, X.-X. Wu, X.-L. Peng, T. Qian, S.-C. Wang, J.-P. Hu, Y.-J. Sun, and H. Ding, FeSe ${ }_{1-x} \mathrm{Se}_{x}$ monolayer films: Towards the realization of high-temperature connate topological superconductivity, Sci. Bull. 62, 503 (2017).

[54] C.-C. Liu, H. Jiang, and Y. Yao, Low-energy effective Hamiltonian involving spin-orbit coupling in silicene and twodimensional germanium and tin, Phys. Rev. B 84, 195430 (2011).

[55] D. Liu, W. Zhang, D. Mou, J. He, Y.-B. Ou, Q.-Y. Wang, Z. Li, L. Wang, L. Zhao, S. He, Y. Peng, X. Liu, C. Chen, L. Yu, G. Liu, X. Dong, J. Zhang, C. Chen, Z. Xu, J. Hu et al. Electronic origin of high-temperature superconductivity in single-layer FeSe superconductor, Nat. Commun. 3, 931 (2012).

[56] Q. Fan, W. H. Zhang, X. Liu, Y. J. Yan, M. Q. Ren, R. Peng, H. C. Xu, B. P. Xie, J. P. Hu, T. Zhang, and D. L. Feng, Plain $s-$ wave superconductivity in single-layer FeSe on $\mathrm{SrTiO}_{3}$ probed by scanning tunnelling microscopy, Nat. Phys. 11, 946 (2015).

[57] C. Fang, Y.-L. Wu, R. Thomale, B. A. Bernevig, and J. $\mathrm{Hu}$, Robustness of $s$-Wave Pairing in Electron-Overdoped $A_{1-y} \mathrm{Fe}_{2-x} \mathrm{Se}_{2}(a=\mathbf{K}, \mathrm{Cs})$, Phys. Rev. X 1, 011009 (2011).
[58] Y. Zhou, D.-H. Xu, F.-C. Zhang, and W.-Q. Chen, Theory for superconductivity in $(\mathrm{Tl}, \mathrm{K}) \mathrm{Fe}_{x} \mathrm{Se}_{2}$ as a doped Mott insulator, Europhys. Lett. 95, 17003 (2011).

[59] F. Yang, F. Wang, and D.-H. Lee, Fermiology, orbital order, orbital fluctuations, and cooper pairing in iron-based superconductors, Phys. Rev. B 88, 100504(R) (2013).

[60] G. R. Stewart, Superconductivity in iron compounds, Rev. Mod. Phys. 83, 1589 (2011).

[61] P. J. Hirschfeld, M. M. Korshunov, and I. I. Mazin, Gap symmetry and structure of Fe-based superconductors, Rep. Prog. Phys. 74, 124508 (2011).

[62] I. I. Mazin, Symmetry analysis of possible superconducting states in $\mathrm{K}_{x} \mathrm{Fe}_{y} \mathrm{Se}_{2}$ superconductors, Phys. Rev. B 84, 024529 (2011).

[63] Z. P. Yin, K. Haule, and G. Kotliar, Spin dynamics and orbitalantiphase pairing symmetry in iron-based superconductors, Nat. Phys. 10, 845 (2014).

[64] J. Hu, Iron-Based Superconductors as Odd-Parity Superconductors, Phys. Rev. X 3, 031004 (2013).

[65] Y. Zhang, J. J. Lee, R. G. Moore, W. Li, M. Yi, M. Hashimoto, D. H. Lu, T. P. Devereaux, D.-H. Lee, and Z.-X. Shen, Superconducting Gap Anisotropy in Monolayer FeSe Thin Film, Phys. Rev. Lett. 117, 117001 (2016).

[66] D. F. Agterberg, T. Shishidou, J. O’Halloran, P. M. R. Brydon, and M. Weinert, Resilient Nodeless $d$-Wave Superconductivity in Monolayer FeSe, Phys. Rev. Lett. 119, 267001 (2017).

[67] Z. Ge, C. Yan, H. Zhang, D. Agterberg, M. Weinert, and $\mathrm{L}$. Li, Evidence for $d$-wave superconductivity in single layer $\mathrm{FeSe} / \mathrm{SrTiO}_{3}$ probed by quasiparticle scattering off step edges, Nano Lett. 19, 2497 (2019).

[68] T. A. Maier, S. Graser, P. J. Hirschfeld, and D. J. Scalapino, $d$-wave pairing from spin fluctuations in the $\mathrm{K}_{x} \mathrm{Fe}_{2-y} \mathrm{Se}_{2}$ superconductors, Phys. Rev. B 83, 100515(R) (2011).

[69] F. Wang, F. Yang, M. Gao, Z.-Y. Lu, T. Xiang, and D.-H. Lee, The electron pairing of $\mathrm{K}_{x} \mathrm{Fe}_{2-y} \mathrm{Se}_{2}$, Europhys. Lett. 93, 57003 (2011).

[70] X.-J. Liu, C. L. M. Wong, and K. T. Law, Non-Abelian Majorana Doublets in Time-Reversal-Invariant Topological Superconductors, Phys. Rev. X 4, 021018 (2014).

[71] Z. Zhang, Y.-H. Wang, Q. Song, C. Liu, R. Peng, K. Moler, D. Feng, and Y. Wang, Onset of the meissner effect at $65 \mathrm{~K}$ in FeSe thin film grown on Nb-doped $\mathrm{SrTiO}_{3}$ substrate, Sci. Bull. 60, 1301 (2015).

[72] L. Zhao, A. Liang, D. Yuan, Y. Hu, D. Liu, J. Huang, S. He, B. Shen, Y. Xu, X. Liu, L. Yu, G. Liu, H. Zhou, Y. Huang, X. Dong, F. Zhou, K. Liu, Z. Lu, Z. Zhao, C. Chen et al., Common electronic origin of superconductivity in $(\mathrm{Li}, \mathrm{Fe}) \mathrm{OHFeSe}$ bulk superconductor and single-layer $\mathrm{FeSe} / \mathrm{SrTiO}_{3}$ films, Nat. Commun. 7, 10608 (2016).

[73] Z. F. Wang, H. Zhang, D. Liu, C. Liu, C. Tang, C. Song, Y. Zhong, J. Peng, F. Li, C. Nie, L. Wang, X. J. Zhou, X. Ma, Q. K. Xue, and F. Liu, Topological edge states in a high-temperature superconductor $\mathrm{FeSe} / \mathrm{SrTiO}_{3}(001)$ film, Nat. Mater. 15, 968 (2016).

[74] G. Kresse and J. Hafner, Ab initio molecular dynamics for liquid metals, Phys. Rev. B 47, 558 (1993).

[75] N. Marzari, A. A. Mostofi, J. R. Yates, I. Souza, and D. Vanderbilt, Maximally localized Wannier functions: Theory and applications, Rev. Mod. Phys. 84, 1419 (2012). 
[76] M. P. L. Sancho, J. M. L. Sancho, and J. Rubio, Quick iterative scheme for the calculation of transfer matrices: Application to $\mathrm{Mo}(100)$, J. Phys. F 14, 1205 (1984).

[77] M. P. L. Sancho, J. M. L. Sancho, J. M. L. Sancho, and J. Rubio, Highly convergent schemes for the calculation of bulk and surface green functions, J. Phys. F 15, 851 (1985).
[78] Q. Wu, S. Zhang, H.-F. Song, M. Troyer, and A. A. Soluyanov, WannierTools: An open-source software package for novel topological materials, Comput. Phys. Commun. 224, 405 (2018).

[79] M. B. Salamon, N. Cornell, M. Jaime, F. F. Balakirev, A. Zakhidov, J. Huang, and H. Wang, Upper critical field and Kondo effects in $\mathrm{Fe}\left(\mathrm{Te}_{0.9} \mathrm{Se}_{0.1}\right)$ thin films by pulsed field measurements, Sci. Rep. 6, 21469 (2016). 